\title{
Ink-Jet Printed Conducting Polyaniline based Flexible Humidity Sensor
}

\author{
Milind V. Kulkarni ${ }^{*}$, Sanjay K. Apte, Sonali D. Naik, Jalindar D. Ambekar and \\ Bharat B. Kale \\ Nanocomposite Laboratory, \\ Centre for Materials for Electronics Technology (C-MET), \\ Department of Information Technology, Govt. of India \\ Panchawati, Off Pashan Road, \\ Pune 411 008, INDIA \\ milindcmet@yahoo.com \\ milind@cmet.gov.in
}

\begin{abstract}
Ink-Jet Printed, Intrinsically Conducting Polymer (ICP), polyaniline have been used for humidity sensing at room temperature. Polyaniline based, aqueous ink-jet printable ink has been synthesized by single step, chemical oxidative polymerization technique. Sulphonic acids were used as a dopant during the in-situ polymerization process. This is a single step polymerization process for the direct synthesis of conducting emeraldine salt phase of the polymer as an ink formulation. The synthesized polyaniline ink was further characterized by spectroscopic (UV-Vis. and FT-IR) analysis which confirmed the presence of conducting emeraldine salt phase of the polymer. The viscosity of the ink was measured by using Brook-field viscometer. The successive trials were performed for the printing of IDT pattern on the flexible, untreated polymer substrate using HP ink-jet- printer. The printed sensor was subjected for the humidity sensing measurements. The change in the resistance with change in the $\% \mathrm{RH}$ was observed. It is suggested that the increase in conductivity at high humidity may be related to a vapour-induced change in the transfer of charge carriers between the polymer chains. The synthesized polyaniline based ink can also be considered as a good candidate for variety of ink-jet printed low cost electronics devices.
\end{abstract}

Keywords: Conducting Polyaniline, Chemical Synthesis, Spectroscopy, Ink-jet Printing, Flexible Sensor

\section{Introduction}

Manufacture of electric circuits on polymer substrates is broadly referred to as flexible electronics and has gained significant interest as a pathway to low-cost or large-area electronics [1,2]. Although conventional vacuum deposition and photolithographic patterning methods are well developed for inorganic microelectronics, they are not appropriate for this application. Flexible polymer substrates are chemically incompatible with resists, etchants, and developers used in conventional integrated circuit (IC) processing. In practice, the usual IC fabrication processes involve multiple steps and high processing temperatures and produce toxic waste, all of which add to their cost. Furthermore, the increasing size of electronic devices such as displays poses great difficulty in adapting standard micro fabrication techniques, including lithographic patterning.
Devices based on organic semiconductors are considered to be very promising for these applications since they may potentially be fabricated entirely using printing technologies, eliminating the need for such major costs as in lithography, vacuum processing including physical vapour deposition, plasma etching, and chemical vapour deposition (CVD), while simultaneously allowing the use of reel-to-reel processing, resulting in reduced substrate handling and clean-room costs as well. Furthermore, since printing is inherently additive in nature, material and disposal costs are also expected to be reduced, resulting in an extremely low net system cost. To print electronics, conducting ink is a key factor for the proper performance of printed electronics. Conductive polymers are currently being developed for a range of different applications, such as chemical sensors, displays and 'plastic' 
transistors [3-5]. Inkjet printing is now an important technology for depositing layers of conductive polymers [6,7]. The method works by ejecting an ink through very fine nozzles. The nozzle diameters of recent thermal or piezoelectric inkjet printers decreased to $3 \mu \mathrm{m}$ for better printing resolutions. Also, electrohydrodynamic inkjet printers can use a submicron size nozzle to print dots with diameters smaller than $1 \mu \mathrm{m}$ [8-10]. The advantages of inkjet printing over other thin film techniques lie in its patterning capability, the efficient use of material, the high speed and low cost of the process, and in the fact that thin films can be printed on flexible substrates. For chemical sensing applications, the more 'open' morphology of inkjet printed films (i.e. a series of connected droplets) may allow rapid diffusion of the water vapour molecules into and out of the film, leading to fast response and recovery times.

Among organic conducting polymers, polyaniline (Pani) is regarded as one of the most technologically promising electrically conductive polymers due to its ease of synthesis, low cost, versatile processability and relatively stable electrical conductivity.

Industrial processes and human comfort, both, need to measure and control the humidity in the environment. In recent years, the need of humidity sensor has greatly increased because of its applications; in control of air conditioning, quality control of food products in a wide range of industries, paper and textile industries, optimal functioning of modern solid state electronic equipment, civil engineering etc. The operating conditions and requirements of humidity sensors depend on the field of application. Therefore, development of low cost humidity sensors with better specifications is required.

In this report, we would like to present the direct synthesis of inkjet printable conducting polyaniline based ink via a single-step, in situ polymerization process using sulfonic acids as a dopant. Also, herein we report the physicochemical characterization of the developed ink, its successive printing of interdigitated (IDT) pattern on flexible, untreated polymeric substrates and its utilization as a flexible, printed humidity sensor.

\section{Experimental details}

All chemicals used were of analytical reagent (AR) grade and the solutions were prepared in doubly distilled water. The polymerization of the monomer, aniline (1 M) was initiated by the dropwise addition of the oxidizing agent, Ammonium persulphate $(1 \mathrm{M})$ under constant stirring at low temperature between $0-5^{0} \mathrm{C}$ in an acidified solution containing $1.33 \mathrm{M}$ organic sulfonic acids. The monomer to oxidizing agent ratio was kept as 1:1. After dropwise addition of oxidizing agent, the reaction mixture slowly turned slightly pink and then converted to a dark green colour after complete addition of the oxidizing agent under constant stirring for $24 \mathrm{~h}$. The dark green coloured aqueous suspension of the polymer was further subjected to physicochemical characterization. UV-Vis. spectra of the polymer solution were recorded by using a Hitachi-U3210 spectrophotometer in the range of 300-900 nm. FT-IR spectra of the polymer were taken on a Perkin-Elmer Spectrum 2000 spectrophotometer between 400 and $4000 \mathrm{~cm}^{-1}$.

A commercial Hewlett-Packard (HP) thermal printer (DeskJet 693C) with a resolution of 600 $X 600$ dots per inch was used in this paper. The only modification to the equipment was to replace the original ink with the polyaniline ink solution. The printer cartridges were emptied and thoroughly rinsed with pure water and acetone to ensure that, there was no ink left in the reservoir. The perfectly cleaned cartridge was then refilled with the developed polyaniline based aqueous ink and the IDT pattern was printed on untreated polymeric substrates.

The humidity sensing measurements were performed by subjecting the printed flexible sensor to dynamic humidity measurements; details about the humidity chamber and measurements have been published in our earlier paper [11]

\section{Results and discussion}

Figure 1 shows the dark green polyaniline based aqueous ink (a) and printed IDT pattern on the flexible, untreated polymer substrate (b), (c). In the present investigation we have used an untreated OHP transparency (crystal clear polyester sheet) for printing the IDT pattern. We also have performed a 'Scotch tape test' to check the adhesion of the ink to the substrate by applying the tape and peeling back by a rapid pull force applied approximately perpendicular (right angle) to the test area. The adhesion of the developed ink was found to be good on the polymer (transparency) sheet.

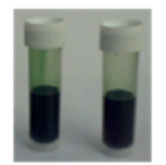

(a)

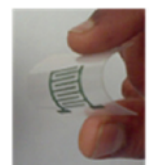

(b)

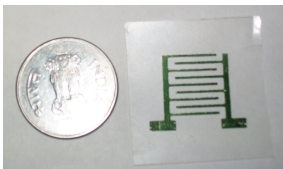

(c)
Figure 1. Polyaniline ink (a) and Ink Jet printed IDT pattern on flexible polymer substrate (b), (c). 
The UV-Visible absorption spectrum of the polyaniline based aqueous ink is presented as Figure 2.

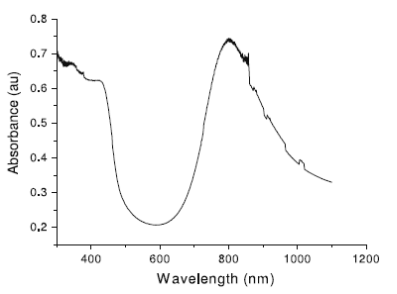

Figure 2. UV-Vis. absorption spectrum of polyaniline ink.

The optical absorption spectrum exhibits a peak at $320 \mathrm{~nm}$ together with a shoulder at $420 \mathrm{~nm}$ and a very sharp peak at $820 \mathrm{~nm}$ with an extended free carrier tail at higher wavelength. The peak at $320 \mathrm{~nm}$ corresponds to the $\pi-\pi^{*}$ transition of the benzenoid rings, while a small peak at $420 \mathrm{~nm}$ can be attributed to the localized polarons which are the characteristics of the protonated polyaniline. The sharp peak at $820 \mathrm{~nm}$ characteristic of the extended coil conformations assigned to the conducting emeraldine salt phase of the polymer [12].The spectral feature observed in figure 2 reveals efficient doping of the polymer by sulfonic acid and is further supported by FT-IR spectroscopic characterization.

Figure 3 represents the FT-IR spectra of the polyaniline based aqueous ink.

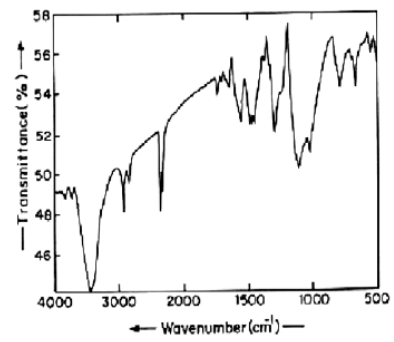

Figure 3. FT-IR Spectrum of polyaniline ink

The peak at $809.02 \mathrm{~cm}^{-1}$ is assigned to the Para disubstituted aromatic rings indicating polymer formation. $\mathrm{C}-\mathrm{H}$ out of plane and in plane bending vibration appears at $\sim 601.53$ and $1121.54 \mathrm{~cm}^{-1}$ respectively. The peak at 1020.76 $\mathrm{cm}^{-1}$ is due to $\mathrm{SO}^{-}$group of the sulphonic acids. Aromatic $\mathrm{C}-\mathrm{N}$ stretching indicating a secondary aromatic amine group appears at $1328.60 \mathrm{~cm}^{-1}$. The $>\mathrm{N}-\mathrm{H}$ stretching vibration modes shows its presence as a peak at $3438.54 \mathrm{~cm}^{-1}$. The two sharp peaks at 1498.15 and $1587.49 \mathrm{~cm}^{-1}$ represents the $\mathrm{C}-\mathrm{N}$ stretching vibrations. The higher frequency vibration at $1600 \mathrm{~cm}^{-1}$ has a major contribution from the quinoid rings while the lower frequency mode at $1500 \mathrm{~cm}^{-1}$ depicts the presence of benzenoid ring units. The presence of these two bands clearly shows that the polymer is composed of the amine and imine units. Further, this also supports our UV-Vis characterization, discussed earlier, where the different phases are observed in the spectrum. The presence of vibration bands of the dopant ion and other characteristic bands confirm the presence of a conducting emeraldine salt phase in the polymer.

After successful synthesis, characterization and inkjet printing of the polyaniline based ink, we have tested its application as a flexible, humidity sensor. The printed polyaniline based sensor was then subjected to the different percentage of relative humidity (\%RH) atmosphere and studied as a sensor for humidity. From the results, it is observed that the printed ink responds to the change in humidity (\% RH atmosphere) by undergoing a change in its resistance. Figure 4 shows the typical response curves for the polyaniline ink subjected to the different humidity atmospheres ranging between 20 and $100 \% \mathrm{RH}$.

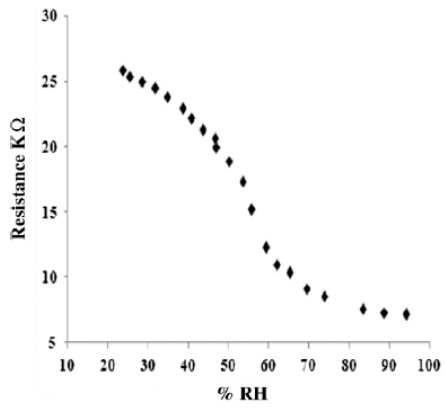

Figure 4. Humidity sensing response of inkjet printed polyaniline ink on untreated flexible polymeric substrate.

From the figure it is observed that, the resistance is seen to be decreased on increasing the $\%$ relative humidity $(\% \mathrm{RH})$ of the polyaniline based printed sensor (i.e. the conductivity is found to be increased with increase in humidity). The resistance is found to be decreased almost linearly from 20 to $80 \%$ $\mathrm{RH}$. From $80 \% \mathrm{RH}$, the decrease in resistance is very small and found to be saturated up to $100 \% \mathrm{RH}$. This clearly indicates that the sensor shows best sensing response from 20 to $80 \%$ $\mathrm{RH}$. The decrease in the resistance or increase in the conductivity with increasing humidity can be attributed to the mobility of the dopant ion which is loosely attached to the polymer chain by weak van der Waals forces of attraction. At low humidity, the mobility of the dopant ion is restricted because under dry conditions the polymer chains would tend to curl up into compact coil form. On the contrary, at high humidity, the polymer absorbs water molecules, and the polymer chains get hydrated. As a result, swelling up of the polymer chains takes 
place, followed by the uncurling of the compact coil form into straight chains that are aligned with respect to each other. This geometry of the polymer is favourable for enhanced mobility of the dopant ion or the charge transfer across the polymer chains and hence the conductivity. Furthermore, the adsorbed water molecules or water content also plays an important role in the transport properties. It has been reported that the conductivity of conducting polymer increases when the sample absorbs the moisture. Decrease of resistance with increase in the humidity proves the adsorption of the water molecules, which makes the polymer more p-type in nature, i.e. the hole concentration is increased by donation of the lone pair from the conducting complex towards the water molecules. Thus, the partial charge transfer process of conducting species with that of water molecules results in the decrease in the sheet resistivity. The detailed mechanism of the humidity sensing by polyaniline is explained in our earlier publication [13]. The almost linear variation with respect to \% $\mathrm{RH}$ can be used in an amplifier circuit for converting the measured values into measurable $\% \mathrm{RH}$ values.

Further detailed investigations of hysteresis, stability and shelf life of the sensor are in progress in our laboratory. Also, we are working on the fabrication of miniaturized sensor device.

\section{Conclusions}

This work demonstrates the single-step, in-situ chemical polymerization process for the direct synthesis of aqueous, conducting polyaniline based ink and its spectroscopic characterization. The developed ink was then successfully utilized for printing an IDT pattern using an inkjet printer on a flexible, untreated polymeric substrate and used as a sensor for humidity. The advantage of using inkjet print technology is the high speed and low cost of fabrication as well as the possibility of printing onto flexible substrates. This conducting polyaniline based aqueous ink will also have potential applications in low cost RFID tags, polymer photovoltaic cells and in printed, low cost flexible electronics devices like Thin film transistors (TFTs) and Organic Light Emitting Diodes (OLEDs).

\section{Acknowledgments}

The authors would like to thank DIT, New Delhi for financial assistance and $\mathrm{Dr} D$. $P$. Amalnerkar, Executive Director, C-MET, for constant encouragement.

\section{References}

[1] S. H. Ko, J. Chung, H. Pan, C. P. Grigoropoulos, and D. Poulikakos, Fabrication of multilayer passive and active electric components on polymer using inkjet printing and lowtemperature laser processing, Sens. Actuators A: Phys. 134, 161-168 (2007); doi:10.1016/j.sna.2006.04.036

[2] S. H. Ko, H. Pan, C. P. Grigoropoulos, C. K. Luscombe, J. M. J. Fr'echet, and D. Poulikakos, All-inkjet-printed flexible electronics fabrication on a polymer substrate by lowtemperature high-resolution selective laser sintering of metal nanoparticles, Nanotechnology, 18,3452022007; doi:10.1088/0957-4484/18/34/345202

[3] L. M. Leung, C. F. Kwong, C. C. Kwok and S. K. So, Organic polymer thick film light emitting diodes (PTF-OLED), Displays, 21,199201(2000); doi: 10.1016/S0141-9382(00)000524

[4] S. R. Forrest, The path to ubiquitous and low coat organic electronic appliances on plastic Nature, 428, 911-918 (2004); doi: 10.1038/nature02498

[5] W. A. MacDonald, Engineered films for display technologies J. Mater. Chem. 14, 4-10 (2004); doi: 10.1039/B310846P

[6] M. Yoshida, S. Uemura, T. Kodzasa, H. Ushijima and T. Kamata, High performance organic FET with double-semiconductor layers Synth. Met. 137, 893-894 (2003); doi:10.1016/S03796779(02)01129-3

[7] K. E. Paul, W. S. Wong, S. E. Ready and R. A. Street, Additive jet printing of polymer thin film transistor Appl. Phys. Lett. 83, 2070-2072 (2003); doi: 10.1063/1.1609233

[8] P. Calvert, Ink jet printing for materials and devices, Chem. Mater. 13, 3299-3305 (2001); doi: $10.1021 / \mathrm{cm} 0101632$

[9] J. U. Park, High-resolution electrohydrodynamic jet printing, Nature Mater. 6, 782-789 (2007); doi: $10.1038 / \mathrm{nmat} 1974$

[10] J. U. Park, J. H. Lee, U. Paik, Y. Lu and J. A. Rogers, Nanoscale patterns of oligonucleotides formed by electrohydrodynamic jet printing with applications in biosensing and nanomaterials assembly, Nano Lett. 8, 4210-4216 (2008);doi: $10.1021 / \mathrm{nl} 801832 \mathrm{v}$

[11] M. V. Kulkarni and A. K. Viswanath, Spectroscopic, thermal and electrical properties of sulphonic acids doped poly(o-anisidine) and their application as humidity sensor, Sens. and Actuators B: chem., 107, 791-797 (2005); doi: 10.1016/j.snb.2004.12.019

[12] M. V. Kulkarni, A. K. Viswanath, R. Marimuthu and T. Seth, Synthesis and characterization of polyaniline doped with organic acids' J. Polym. Sci. A 42, 2043-2049 (2004); doi: 10.1002/pola.11030

[13] M. V. Kulkarni and A. K. Viswanath, Studies on sulphonic acids doped poly ( $\mathrm{N}$-ethyl aniline): a material for humidity sensing application, Polym. Eng. Sci. 47, 1621-1629(2007); doi: 10.1002/pen.2083 